\title{
Forschungsnotiz
}

\section{Gesellschaftliche Machtverhältnisse und Einkommensverteilung: Ein kritischer Replikationsversuch zu einer Studie von Bornschier und Ballmer-Cao}

\author{
Erich Weede, Horst Tiefenbach \\ Universität zu Köln, Forschungsinstitut für Soziologie \\ Lindenburger Allee 15, D-5000 Köln 41
}

Z u s a m m e n f a s s u n g: Für Lenski (1973) ist die Machtverteilung die entscheidende Determinante der Privilegien- und Einkommensverteilung. Bornschier und Ballmer-Cao $(1979,1980)$ haben diesen Grundgedanken in einen international vergleichenden Untersuchungsplan umgesetzt. Landkonzentration, Drohstärke der Lohnabhängigen, Bürokratisierung und Staatsquote sind ihre Indikatoren der Machtverteilung und ihre Determinanten der Einkommensverteilung. In der vorliegenden Studie wird gezeigt, daß weder Bürokratisierung noch Staatsquote nennenswert mit der Einkommensverteilung zusammenhängen, wenn das ökonomische Entwicklungsniveau und die Humankapitalausstattung von Gesellschaften kontrolliert werden. Auch der Beitrag der Drohstärke der Lohnabhängigen zur Einkommensverteilung wird hier nicht repliziert. Sofern es einen Machtindikator gibt, der zur Egalisierung der Einkommensverteilung beiträgt, ist es der militärische Partizipationsgrad.

\section{Einleitung}

Ausgangspunkt der folgenden Uberlegungen ist ein Gedanke von Gerhard Lenski (1973: 71-72): „Wenn Privilegien definiert sind als Besitz oder Kontrolle eines Teils des Surplus, den eine Gesellschaft produziert, dann folgt daraus, daß Privilegien weitgehend eine Funktion von Macht und in einem sehr geringen Maß eine Funktion von Altruismus sind. Die Verteilung von Privilegien in einer Gesellschaft zu erklären, heißt also vornehmlich die Verteilung von Macht aufzudecken." Danach sind machtbedingte Durchsetzungschancen die Hauptdeterminante von Privilegien, einschließlich mehr oder weniger privilegierter Einkommen.

Bornschier und Ballmer-Cao $(1979,1980)$ haben diesen Gedanken Lenskis in einer international vergleichenden Studie aufgegriffen. Dort haben sie auch Indikatoren für die Machtverteilung vorgeschlagen, nämlich: Bürokratisierung, die als Anteil der Büroangestellten an der $\mathrm{Ge}$ samtzahl der außerhalb der Landwirtschaft Beschäftigten operationalisiert wird; Landkonzentration, die als Gini-Index der landwirtschaftlichen Bodenverteilung operationalisiert wird; Machtverteilung auf dem Arbeitsmarkt oder auch „Drohstärke der Lohnabhängigen“, die über die Durchschnit tsbeteiligung bei Lohn- und Arbeitskonflikten in einer Fünfjahresperiode erfaßt wird; Staatsquote, d.h. Anteil der Regierungseinnahmen am Bruttoinlandsprodukt. Nach Bornschier und Ballmer-Cao (1979: 501; 1980: 232) sind Einkommen und Macht in einer Gesellschaft um so egalitärer verteilt, je stärker das Ausmaß der Bürokratisierung, je geringer die Landkonzentration, je größer die Drohstärke der Lohnabhängigen und je größer die Staatsquote ist. Multiple Regressionen für verschiedene Indikatoren der Einkommensverteilung zum Ende der 60er Jahre und für 50 Gesellschaften sollen diese Auffassung stützen.

Der Versuch von Bornschier und Ballmer-Cao $(1979,1980)$, die Machtverteilung zu erfassen, kann rein theoretisch kritisiert werden. Zwar ist es plausibel, daß Landbesitzkonzentration eine ungleiche Verteilung von Macht und Einkommen bedingt, nicht aber - wie von den multiplen Regressionen Bornschiers und Ballmer-Caos $(1979,1980)$ impliziert daß der Effekt der Landkonzentration auf die Einkommensverteilung unabhängig vom Agraranteil am Sozialprodukt in allen Gesellschaften gleich stark ist. $\mathrm{Zu}$ erwarten ist vielmehr, daß Konzentration des Landbesitzes vor allem bei agrarisch geprägten Volkswirtschaften die Ungleichheit der Einkommensverteilung erhöht.

Auch bei der Staatsquote ist nicht einzusehen, daß hohe Werte auf eine egalitäre Machtverteilung - und folglich auch Einkommensverteilung - hinauslaufen. Eine hohe Staatsquote kann ja die Konzentration politischer und ökonomischer Macht in den Händen einer kleinen Zahl von Politikern und Bürokraten bedeuten, die vielleicht aber eine ganz andere Einkommenspolitik verfolgen. Unabhängig davon, ob die 
Staatsquote tatsächlich positiv mit der Einkommensverteilung zusammenhängt - es gibt erheblichen Anlaß zu Zweifeln (Weede 1980; Weede und Tiefenbach 1981a) - erscheint uns die theoretische Begründung des Zusammenhangs nicht zwingend.

\section{Warum ,Bürokratisierung' als steigender Anteil} der Beschäftigten in Büroberufen zur Egalisierung der Einkommensverteilung beitragen soll, können wir nicht nachvollziehen ${ }^{1}$. Dies könnte der Fall sein, wenn Büroangestellte im allgemeinen höher qualifiziert, schwerer ersetzbar, in diesem Sinne mächtiger und besser bezahlt wären als andere Arbeitnehmer, wenn außerdem schon ein großer Teil der Beschäftigten im Büro arbeitete. Falls nur die erste, nicht aber die zweite Bedingung gilt, könnte Bürokratisierung zumindest in frühen Entwicklungsphasen bzw. auf niedrigem Entwicklungsniveau auch zur Ungleichheit der Einkommen beitragen. Zweifel am egalisierenden Effekt von Bürokratisierung sind also theoretisch begründbar. Mit Bürokratisierung eng zusammenhängen dürfte jedoch das Wachstum des tertiären Sektors. Nach Evans und Timberlake (1980) erhöht dieses allerdings die Ungleichheit der Einkommensverteilung ${ }^{2}$. Damit wird die Frage aufgeworfen, ob jede Art der Bürokratisierung immer zur Egalisierung der Einkommen beiträgt.

Warum nur die durchschnittliche Streikbeteiligung, nicht aber Häufigkeit oder Dauer oder Streikvolumen (als Produkt von Häufigkeit, Dauer und Beteiligung) oder auch der gewerkschaftliche Organisationsgrad die ,Drohstärke der Lohnabhängigen" erfassen, ist zumindest ein Problem, das einer weitergehenden theoretischen Elaboration bedarf. Außerdem muß auch bezweifelt werden, ob gewerkschaftliche Organisation und ,Drohstärke der Lohnabhängigen' zur Egalisierung der Einkommen beitra-

1 Bornschiers (1981a: 120, Anmerkung 7) jüngster Beitrag zur ,Arbeitsteilung, strukturellen Mobilität und Klassenbildung" trägt auch nicht zur Klärung bei, eher zum Gegenteil.

2 Die Studie von Evans und Timberlake (1980) leidet allerdings darunter, daß der kurvilineare Zusammenhang zwischen dem Bruttosozialprodukt pro Kopf und der Einkommensverteilung nicht richtig spezifiziert worden ist. Empirisch belegen kann sie wegen dieses Fehlers gar nichts mehr, nur noch Möglichkeiten andeuten. gen. Denkbar ist auch, daß Gewerkschaften die Reallöhne mancher Arbeitnehmer auf Kosten anderer Arbeitnehmer oder um den Preis der Arbeitslosigkeit erhöhen (Hayek 1971: 344; McKenzie und Tullock 1978: 273), also zur Ungleichheit der Einkommensverteilung beitragen. Die Zweifel daran, ob Bornschier und Ballmer-Cao $(1979,1980)$ wirklich zentrale Aspekte der Machtverteilung und Determinanten der Einkommensverteilung angemessen erfaßt haben, sind also teilweise theoretischer Natur. Diese Zweifel wollen wir zunächst zuriickstellen und uns der Frage zuwenden, ob die Ergebnisse von Bornschier und Ballmer-Cao $(1979,1980)$ ohne weiteres replizierbar sind.

\section{Ein Replikationsversuch}

Der folgende Replikationsversuch bezieht sich auf dieselben 50 Gesellschaften und weitgehend dieselben Daten wie die Studie von Bornschier und Ballmer-Cao (1979). Datenquelle für den Gini-Index, die Einkommensanteile ${ }^{3}$ der oberen $20 \%$ und der unteren $60 \%$ ist ebenso wie für die unabhängigen Variablen Bürokratisierung, Landkonzentration, Drohstärke der Lohnabhängigen und Staatsquote das Handbuch von Ballmer-Cao und Scheidegger (1979), herausgegeben von Bornschier und Heintz.

Zweck des Replikationsversuchs ist nicht etwa die Suche nach Rechenfehlern, sondern zu untersuchen, ob scheinbar geringfügige Veränderungen des Untersuchungsplans größere inhaltliche Implikationen haben können. Nach unseren Erfahrungen (Weede und Tiefenbach 1981b) passiert das gerade bei der vergleichenden Analyse von Einkommensverteilungen nur allzu leicht. Was wir anders als Bornschier und Ballmer-Cao $(1979,1980)$ gemacht haben, ist folgendes:

1. Während Bornschier und Ballmer-Cao (1979, 1980) versucht haben, die Werte für die unabhängen Variablen möglichst auf das gleiche Jahr

3 Die Verteilungsdaten beziehen sich meist auf Haushalte. Sie erfassen Einkommen vor Steuern. Näheres bei Ballmer-Cao und Scheidegger (1979: $212 \mathrm{ff}$.). 
wie bei den abhängigen $z u$ beziehen ${ }^{4}$, haben wir uns bemüht, die unabhängigen Variablen möglichst dicht an $1965 \mathrm{zu}$ erfassen. Sowohl Bornschier und Ballmer-Cao als auch wir versuchen mit unseren Entscheidungen, das Problem einer unzulänglichen Datenbasis zu bewältigen, d.h. damit fertig zu werden, daß vorhandene Einkommensverteilungsdaten sich auf verschiedene Jahre beziehen, wenn auch eine gewisse Häufung in den späten 60er Jahren festgestellt werden kann. Bornschier und Ballmer-Cao wollen gleichzeitige Messung der unabhängigen und abhängigen Variablen auch um den Preis erreichen, daß sich Korrelationen und Regressionen auf eine von Land zu Land schwankende Jahresbasis beziehen. Wir wollen möglichst alle Messungen ca. 1965 vornehmen, auch um den Preis, daß sich viele Verteilungsmessungen dann auf einen anderen Zeitpunkt als den der mutmaßlichen Bestimmungsgründe beziehen. Beide Strategien sind Notlösungen. Wir wollen nicht behaupten, daß unser Untersuchungsplan besser sei als der von Bornschier und Ballmer-Cao $(1979,1980)$, sondern nur, daß er nicht schlechter ist und daß man Ergebnissen mißtrauen sollte, die mit dem jeweils anderen Untersuchungsplan nicht replizierbar sind.

\section{Bornschier und Ballmer-Cao $(1979,1980)$ ha-} ben bei Landkonzentration und Drohstärke der Lohnabhängigen ihre ursprünglich quantitativen Variablen unter Informationsverlust in ranggeordnete Kategorien verwandelt, um die Werte von Ländern mit fehlenden Datenangaben besser schätzen zu können. Wir werden stattdessen auf solche Rekodierungen mit Informationsverlust verzichten und dafür eher einige fehlende Datenangaben bei den unabhängigen Variablen hinnehmen, zumal die Angaben von Bornschier und Ballmer-Cao (1979: 504; 1980: 240) Anlaß zu der Hoffnung geben, daß fehlende Datanangaben kein großes Problem sind. Bei der Drohstärke der Lohnabhängigen haben wir die Rohwerte durch den natürlichen Logarithmus ersetzt,

4 Das gilt allerdings nicht für alle Variablen. Beim BSPC haben Bornschier und Ballmer-Cao (1980: 237, Anmerkung 10) freiwillig, bei der Landkonzentration durch die Datenlage gez wungen auf Zeitanpassung verzichtet. Selbst wenn eine durchgehend gelungene Zeitanpassung trotz der Ungleichzeitigkeit nationaler Daten unserem Verfahren vorzuziehen wäre, kann bezweifelt werden, ob das auch noch für eine partielle Zeitanpassung gilt. um die Schiefe von $+5,2$ auf $-0,7$ zu reduzieren ${ }^{5}$.

3. Bornschier und Ballmer-Cao $(1979,1980)$ verwenden füf Indikatoren der Einkommensverteilung: Gini, die Einkommensanteile der obersten $5 \%$, der obersten $20 \%$ (des 5 . Quintils), des 4. Quintils und der untersten $60 \%$. Wir werden im folgenden weder die Einkommensanteile der obersten $5 \%$ noch die des 4 . Quintils berücksichtigen. Gini korreliert $-0,99$ mit dem Einkommensanteil der untersten $60 \%$ und 0,98 mit dem der obersten 20\%. Vom Vorzeichen abgesehen sind sich die auch bei uns verwendeteten Indikatoren der Einkommensverteilung sehr ähnlich ${ }^{6}$. Beim Einkommensanteil der obersten 5\% haben wir größere Zweifel an der Genauigkeit der Schätzung als dort, wo größere Einkommensgruppen behandelt werden. Außerdem korreliert deren Einkommensanteil nur noch $0,90 \mathrm{mit}$ dem Gini-Index, ist also ein weniger gutes $\mathrm{MaB}$ furr die Gleichheit oder Ungleichheit der Einkommensverteilung als die größere Gruppen betreffenden Angaben. Der Einkommensanteil des 4. Quintils korreliert sogar nur noch $-0,71 \mathrm{mit}$ dem Gini-Index. Auch aus konzeptionellen Gründen ist klar, dab der Einkommensanteil des 4. Quintils nicht einfach Gleichheit oder Ungleichheit mißt. Während eine Vergrößerung des Anteils der unteren $60 \%$ oder eine Verringerung des Anteils der oberen 20\% immer mehr Gleichheit impliziert, kann eine Vergrößerung des Anteils des 4. Quintils je nach Ausgangslage mehr oder weniger Ungleichheit als vorher implizieren. Zumindest wirft die Analyse der Einkommensanteile der obersten 5\% und des 4. Quintils Spezialprobleme auf, die wir hier nicht behandeln möchten.

$5 \mathrm{Ob}$ die Rekodierung von Bornschier und BallmerCao auch die Verteilungsschiefe reduzieren sollte, haben wir den Angaben nicht entnommen - vermutlich aber ja.

6 Mancher Leser wird sich fragen, warum wir bei Korrelationen von 0,98 bzw. 0,99 unter unseren Indikatoren überhaupt drei anstelle eines einzigen verwenden. Die Erfahrung bei der Analyse von Verteilungsdaten hat uns gezeigt, daß man auch bei sehr ähnlichen Variablen unter Umständen recht unterschiedliche Ergebnisse erzielt (vgl. vor allem Weede und Tiefenbach 1981b). Mit den drei Ungleichheitsindikatoren ist also ein gewisses Minimum an $\mathrm{Re}$ plikation eingebaut. 
TABELLE 1 Regressionen der Einkommensverteilung auf Indikatoren der Machtverteilung

\begin{tabular}{lccl}
\hline & $\begin{array}{l}\text { 100 mal } \\
\text { Gini }\end{array}$ & $\begin{array}{l}\text { Anteil der } \\
\text { obersten } \\
20 \%\end{array}$ & $\begin{array}{l}\text { Anteil der } \\
\text { untersten } \\
60 \%\end{array}$ \\
\hline Bürokrati- & $-0,73$ & $-0,86$ & 0,56 \\
sierung & 0,10 & 0,04 & 0,10 \\
& $-0,31(-0,34)$ & $-0,36(-0,38)$ & $0,31(0,38)$ \\
Landbesitz- & 0,19 & 0,19 & $-0,14$ \\
konzentra- & 0,04 & 0,02 & 0,05 \\
tion & $0,37(0,33)$ & $0,38(0,33)$ & $-0,35(-0,33)$ \\
Drohstärke & $-1,36$ & $-1,36$ & 1,07 \\
der Lohn- & 0,28 & 0,23 & 0,26 \\
abhängigen & $-0,18(-0,29)$ & $-0,18(-0,27)$ & $0,19(0,26)$ \\
Staats- & $-0,23$ & $-0,27$ & 0,15 \\
quote & 0,20 & 0,10 & 0,27 \\
& $-0,24(-0,22)-0,29(-0,27)$ & $0,21(0,17)$ \\
Konstante & 51,9 & 60,2 & 22,5 \\
& & & \\
bereinigter & & & \\
erklärter & & 42,2 & 26,5 \\
Varianzan- & 30,4 &
\end{tabular}

wo (von den beiden letzten Zeilen abgesehen) in jeder Zelle zuerst der unstandardisierte Regressionskoeffizient, dann dessen Signifikanzniveau, dann der standardisierte Regressionskoeffizient angegeben ist; die in Klammern angegebenen standardisierten $\mathrm{Re}-$ gressionskoeffizienten die bei Bornschier und Ballmer-Cao $(1979,1980)$ in der Schlubregression berichteten Werte sind.

Tabelle 1 stellt die Ergebnisse unseres Versuchs dar, die Schlußregression von Bornschier und Ballmer-Cao (1979: 501; 1980: 232) zu reproduzieren. Ein Vergleich der standardisierten Koeffizienten in unserer Berechnung und in der von Bornschier und Ballmer-Cao zeigt, daß die meisten Koeffizienten eine ähnliche Größenordnung haben. Das gilt bei der ,Drohstärke der Lohnabhängigen" allerdings weniger als anderswo.

Wegen der unterschiedlichen Behandlung des Problems fehlender Datenangaben sind die Signifikanzniveaus hier und bei Bornschier und Ballmer-Cao nicht vergleichbar. Bornschier und Ballmer-Cao haben ja fehlende Angaben explizit geschätzt, während wir dies durch ,pairwise deletion" implizit getan haben, dafur aber in Kauf genommen haben, Signifikanzen auf der Basis von 29 statt $50 \mathrm{zu}$ erhalten. Bei unserem Verfahren ist Signifikanz also wesentlich schwerer zu erreichen als bei dem von Bornschier und
Ballmer-Cao. Dennoch können solche Prädiktoren eindeutig als nicht-bewährt klassifiziert werden, die wie die ,Drohstärke der Lohnabhängigen" nie auch nur das $20 \%-N i v e a u$ erreichen. Nur wer die technischen Entscheidungen von Bornschier und Ballmer-Cao zur Zeitanpassung, zur Rekodierung mit Informationsverlust und zur nirgends berichteten Schätzung fehlender Angaben für die einzig sinnvollen hält, wird weiter diesen Prädiktor als bewährt betrachten können. Uns erscheint es allerdings sinnvoller, inhaltlich soziologischen Aussagen zu mißtrauen, deren Bewährung so stark wie hier von verfahrenstechnischen und teilweise nicht dokumentierten Entscheidungen abhängt.

Die Effekte von Landbesitzkonzentration sind nicht nur ähnlich wie bei Bornschier und Ballmer-Cao, sondern auch durchgehend signifikant. Es ist auch plausibel und unseres Wissens unstrittig, daß Ungleichheiten in Eigentumsverteilungen zu Ungleichheiten in Einkommensverteilungen beitragen. Aus zwei Gründen werden wir dennoch auf die weitere Berücksichtigung dieses bewährten Prädiktors verzichten: Bei Landbesitzkonzentration stehen nur für 37 von 50 Ländern Angaben zur Verfügung. Außerdem ist es theoretisch nicht plausibel, daß die Effekte von Landbesitzkonzentration unabhängig vom Beitrag der Landwirtschaft zum Sozialprodukt sind. Zwar könnte man mit einem Interaktionsterm auch diese Komplikation berücksichtigen, aber dann wuirde das Verhältnis von Beobachtungen und Prädiktoren allzu ungünstig.

Nach den Signifikanzniveaus sind weder die Effekte von Bürokratisierung noch die der Staatsquote überzeugend. Die geringen Signifikanzniveaus können zum Teil durch die geringe Fallzahl (29) und die relative Vielzahl der Prädiktoren entschuldigt werden. Würde man Landbesitzkonzentration und Drohstärke der Lohnabhängigen aus den Regressionen eliminieren und damit auch dem Problem fehlender Angaben fast entgehen (nur bei Bürokratisierung ist $N=49$ statt 50 ), dann würde $z$ war die Signifikanz der Bürokratisierungseffekte fast unverändert (also wie in Tab. 1) bleiben, aber die der Staatsquote würde immer das $2 \%$ und zweimal das $1 \%$-Niveau überspringen. Auch beim standardisierten Regressionskoeffizienten würde sich zeigen, daß die Staatsquote eine bedeutsamere (mutmaßliche) De- 
terminante der Einkommensverteilung ist als Bürokratisierung?

\section{Weiterfiihrende Analysen}

Gegen die sog. Schlußregression von Bornschier und Ballmer-Cao (1979: 501; 1980: 232) und damit auch gegen unsere bisherigen Versuche, diese zu replizieren, lassen sich weitere Einwände erheben. Grundgedanke aller folgenden Einwände ist, daß möglicherweise bedeutsame Prädiktoren der Einkommensverteilung bisher vernachlässigt worden sind und daß ihre Einbeziehung, d.h. die Vermeidung oder Verringerung des Ausmaßes von Spezifikationsfehlern, vorherige Resultate umstoßen könnte. Ein auch bei Bornschier und Ballmer-Cao $(1979,1980)$ stellenweise verwendeter zusätzlicher Prädiktor ist das Bruttosozialprodukt per capita (BSPC), das kurvilinear und nichtmonoton mit der Einkommensverteilung zusammenhängt ${ }^{8}$. Bornschiers und Ballmer-Caos Unterstellung (1979: 502; 1980: 234), daß Bürokratisierung und die Staatsquote zwischen BSPC und der Einkommensverteilung intervenieren, rechtfertigt nicht die Vernachlässigung der Kontrollvariablen BSPC. Möglicherweise werden die Effekte des BSPC nur teilweise von Bürokratisierung und Staatsquote vermittelt; möglicherweise sind die Effekte von Bürokratisierung und Staatsquote bei Einführung von $\mathrm{BSPC}$ als Kontrollvariable nicht mehr signifikant.

Nach Ahluwalia (1976a, 1976b; kritisch: Weede und Tiefenbach 1981b) hängt das Ausbildungsniveau der Bevölkerung bzw. die $\mathrm{Hu}$ mankapitalausstattung der Erwerbspersonen mit der Einkommensverteilung zusammen. Im folgenden werden wir den Einschulungsanteil bei Sekundarschulen relativ zur entsprechenden Altersgruppe als Kontrollvariable ver-

\footnotetext{
7 Das gilt natürlich nur, wenn die zugrunde liegenden Gleichungen angemessen spezifiziert sind. Unsere $Z$ weifel daran werden weiter unten artikuliert.
}

8 Auf den nicht-monotonen Zusammenhang zwischen dem ökonomischen Entwicklungsniveau einerseits und der Einkommensverteilung andererseits hat zuerst Kuznets (1963) hingewiesen. Zum Vergieich alternativer Spezifikationen dieses Zusammenhangs: Weede (1980). wenden?. Diese Variable könnte eng mit Bürokratisierung zusammenhängen, denn Bürotätigkeit setzt im allgemeinen wenigstens halbwegs solide Kenntnisse im Schreiben, Lesen und Rechnen voraus. Vielleicht hat nicht Bürotätigkeit oder Bürokratisierung einen egalisierenden Einkommenseffekt, wie Bornschier und Ballmer-Cao $(1979,1980)$ meinen, sondern das über Sekundareinschulungen erfaßte bessere Ausbildungsniveau der Bevölkerung.

Nach Andreski (1968) ist der militärische Partizipationsgrad eine wichtige Determinante der Macht- und Einkommensverhältnisse in Gesellschaften. Auch Bornschier (1981b, 1981 c) hat sich - allerdings sekptisch - mit dieser Variable auseinandergesetzt. Soweit er den militärischen Partizipationsgrad überhaupt für einen Bestimmungsgrad der Einkommensverteilung hält, betrachtet er - wie auch wir in früheren Schriften (Jagodzinski und Weede 1980; Weede 1981; Weede und Tiefenbach 1981a) - den Militäranteil als Indikator der Machtverhältnisse. Es ist auch plausibel, daß sich bewaffnete Männer schlechter als unbewaffnete Leute ausbeuten lassen.

In Tab. 2 werden die Indikatoren der Einkommensverteilung auf den kurvilinearen Prädiktor BSPC, die Sekundareinschulungsquote, den militärischen Partizipationsgrad, Bürokratisierung und Staatsquote zurückgefuihrt. Betrachten wir die Effekte der Bornschier und Ballmer-Cao $(1979,1980)$ entnommenen Machtvariablen zuerst: Weder Bürokratisierung noch die Staatsquote trägt nennenswert zur Egalisierung der Einkommensverteilung be $i^{10}$. Aber der militärische Partizipationsgrad, der dem theoretischen Denken von Bornschier und Ballmer-Cao (1979, 1980) fremde Indikator der Machtverhältnisse,

9 Datenquelle ist Ballmer-Cao und Scheidegger (1979: 224-226). Diese Daten beziehen sich auf 1960 statt wie die meisten anderen auf ca. 1965. - Daneben haben wir auch den Einschulungsanteil in Primärschulen als Kontrollvariable verwendet. Dieser war allerdings nie signifikant.

10 Würde man den Datensatz über Bornschiers und Ballmer-Caos $(1979,1980) 50$ Länder hinaus soweit wie möglich erweitern ( $\mathrm{N}$ bis $\mathrm{zu} 71$ ), dann blieben die Effekte von Bürokratisierung und Staatsquote immer noch in allen Gleichungen unterhalb des 20\%-Signifikanzniveaus. Das gilt sowohl bei "listwise" als auch bei ,pairwise deletion". 
TABELLE 2 Regressionen der Einkommensverteilung auf Indikatoren der Machtverteilung und Kontrollvariablen

\begin{tabular}{|c|c|c|c|}
\hline & $\begin{array}{l}100 \mathrm{mal} \\
\text { Gini }\end{array}$ & $\begin{array}{l}\text { Anteil der } \\
\text { obersten } \\
20 \%\end{array}$ & $\begin{array}{l}\text { Anteil der } \\
\text { untersten } \\
60 \%\end{array}$ \\
\hline in $B S P C$ & $\begin{array}{r}16,29 \\
0,10\end{array}$ & $\begin{array}{r}17,12 \\
0,07\end{array}$ & $\begin{array}{r}-11,52 \\
0,11\end{array}$ \\
\hline$(\ln \mathrm{BSPC})^{2}$ & $\begin{array}{r}-1,00 \\
0,19\end{array}$ & $\begin{array}{r}-1,05 \\
0,14\end{array}$ & $\begin{array}{l}0,70 \\
0,21\end{array}$ \\
\hline \multicolumn{4}{|c|}{$\begin{array}{l}\text { Beta zu } \\
b_{1}(\ln B S P C) \\
+b_{2} \ln (B S P C)^{2}\end{array}$} \\
\hline & 0,43 & 0,45 & 0,43 \\
\hline $\begin{array}{l}\text { Sekundarein- } \\
\text { schulungs- } \\
\text { quote }\end{array}$ & $\begin{array}{r}-0,29 \\
0,00 \\
-0,77\end{array}$ & $\begin{array}{r}-0,25 \\
0,00 \\
-0,68\end{array}$ & $\begin{array}{l}0,23 \\
0,00 \\
0,81\end{array}$ \\
\hline $\begin{array}{l}\text { militärischer } \\
\text { Partizipa- } \\
\text { tionsgrad } \\
\text { ln (MPR+1) }\end{array}$ & $\begin{array}{r}-2,37 \\
0,04 \\
-0,26\end{array}$ & $\begin{array}{r}-2,05 \\
0,06 \\
-0,23\end{array}$ & $\begin{array}{l}1,79 \\
0,04 \\
0,26\end{array}$ \\
\hline $\begin{array}{l}\text { Bürokrati- } \\
\text { sierung }\end{array}$ & $\begin{array}{l}\text { F-Niveau } \\
\text { unzurej- } \\
\text { chend }\end{array}$ & $\begin{array}{r}-0,28 \\
0,53 \\
-0,12\end{array}$ & $\begin{array}{l}\text { F-Niveau } \\
\text { unzureichend }\end{array}$ \\
\hline $\begin{array}{l}\text { Staats- } \\
\text { quote }\end{array}$ & $\begin{array}{c}-0,055 \\
0,74 \\
-0,06\end{array}$ & $\begin{array}{r}-0,15 \\
0,40 \\
-0,16\end{array}$ & $\begin{array}{l}\text { F-Niveau } \\
\text { unzureichend }\end{array}$ \\
\hline Konstante & $-3,38$ & 3,09 & 62,6 \\
\hline $\begin{array}{l}\text { bereinigter } \\
\text { erklärter } \\
\text { Varianzteil }\end{array}$ & 48,8 & 54,3 & 49,1 \\
\hline
\end{tabular}

wo (von der 3. und den beiden letzten Zeilen abgesehen) in jeder Zelle zuerst der unstandardisierte Regressionskoeffizient, dann dessen Signifikanzniveau, dann der standardisierte Regressionskoeffizient angegeben ist;

ein Signifikanzniveau von 0,00 durch Abrundung eines kleineren Wertes als 0,0049 entstanden ist; der standardisierte Regressionskoeffizient in der 3. Zeile den Einfluß des wirtschaftlichen Entwicklungsniveaus erfafst (vgl. Jagodzinski/Weede 1981 zur Berechnung und Interpretation).

bewährt sich deutlich besser. In drei Gleichungen wird das 5\%-Signifikanzniveau zweimal übersprungen. Würde man Goldbergers (1970) Empfehlung entsprechend die eindeutig nicht signifikanten Prädiktoren Bürokratisierung und Staatsquote eliminieren, dann wären in allen drei Gleichungen die Effekte des militärischen Partizipationsgrades auf dem 5\%-Niveau signifikant.
BSPC und Sekundareinschulungen sind im Kontext dieses Aufsatzes nur Kontrollvariablen. Dennoch muß festgehalten werden, daß nach Tab. 2 die Sekundareinschulungsquote eine entscheidende zur Egalisierung der Einkommensverteilung beitragende Bedingung ist. Vielleicht hängt die Einkommensverteilung entgegen Bornschier und Ballmer-Cao (1979, 1980) nicht primär von den Machtverhältnissen ab, sondern von der Verteilung von Humankapital $^{11}$. Je mehr Schüler Sekundarschulen besuchen, desto egalitärer wird diese Verteilung werden.

$\mathrm{Da}$ der kurvilineare Zusammenhang zwischen BSPC und der Einkommensverteilung hier so wenig ausgeprägt ist und nicht einmal auf dem 5\%-Niveau signifikant, ist ein unerwartetes und unübliches Ergebnis. Grundsätzlich stehen dafür zwei Erklärungen zur Verfügung. Entweder ist unsere Auswahl der 50 Länder untypisch, etwa weil zu wenige ganz arme Länder berück. sichtigt sind und deshalb der kurvilineare $\mathrm{Zu}$ sammenhang nicht mehr aufzeigbar ist, oder die Sekundareinschulungsquote reißt einen großen Teil der Erklärungskraft des BSPC an sich. Dagegen, daß die letztgenannte Interpretation ausreicht, sprechen allerdings die Befunde von $\mathrm{Ah}$. luwalia (1976a, 1976b), wonach der kurvilineare Zusammenhang zwischen BSPC und Einkommensverteilung trotz Einfuihrung von Einschulungsquoten hoch signifikant bleibt.

Es liegt nahe, Tab. 2 durch eine Tab. 3 zu ergänzen, wo gemäß Goldbergers (1970) Empfehlung eindeutig überflüssige Prädiktoren wie Bürokratisierung und Staatsquote eliminiert sind und wo der Datensatz alle greifbaren Informationen berücksichtigt, also zusätzliche

11 Bornschier (1981a: 120) scheint auch die Verteilung von Humankapital letztlich als Resultat der Machtverteilung zu begreifen. Die Herrschenden organisieren Arbeit so, daß die einen Humankapital erwerben können, die anderen aber nicht. Die austauschtheoretische Perspektive impliziert aber eher das Gegenteil, nämlich, daß eine günstige Humankapitalausstattung den Mach terwerb erleichtert. Eine eingehende Diskussion dieser Frage müßte wohl auch die Anlage-Umwelt-Problematik mit einbeziehen und damit den Rahmen dieses Aufsatzes sprengen. 
TABELLE 3 Regressionen der Einkommensverteilung auf BSPC, Sekundareinschulungsquote und militärischen Partizipationsgrad

\begin{tabular}{|c|c|c|c|}
\hline & $\begin{array}{l}100 \mathrm{mal} \\
\text { Gini }\end{array}$ & $\begin{array}{l}\text { Anteil der } \\
\text { obersten } \\
20 \%\end{array}$ & $\begin{array}{l}\text { Anteil der } \\
\text { untersten } \\
60 \%\end{array}$ \\
\hline In BSPC & $\begin{array}{r}27,22 \\
0,00\end{array}$ & $\begin{array}{r}26,01 \\
0,00\end{array}$ & $\begin{array}{r}-20,81 \\
0,00\end{array}$ \\
\hline$(\ln B S P C)^{2}$ & $\begin{array}{r}-1,88 \\
0,01\end{array}$ & $\begin{array}{r}-1,85 \\
0,00\end{array}$ & $\begin{array}{l}1,42 \\
0,01\end{array}$ \\
\hline $\begin{array}{l}\text { Beta } z u \\
b_{1}(\ln B S P C \\
+b_{2}(\ln B S P\end{array}$ & ${ }_{(C)^{2}}^{0,46}$ & 0,38 & 0,51 \\
\hline $\begin{array}{l}\text { Sekundar- } \\
\text { einscliu- } \\
\text { lungsquote }\end{array}$ & $\begin{array}{r}-0,26 \\
0,00 \\
-0,65\end{array}$ & $\begin{array}{r}-0,25 \\
0,00 \\
-0,65\end{array}$ & $\begin{array}{l}0,20 \\
0,00 \\
0,69\end{array}$ \\
\hline $\begin{array}{l}\text { militärischer } \\
\text { Partzipa- } \\
\text { tionsgrad } \\
\text { ln }(\mathrm{MPR}+1)\end{array}$ & $\begin{array}{r}-2,21 \\
0,03 \\
-0,26\end{array}$ & $\begin{array}{r}-1,68 \\
0,07 \\
-0,20\end{array}$ & $\begin{array}{l}1,54 \\
0,04 \\
0,25\end{array}$ \\
\hline Konstante & $-38,2$ & $-26,4$ & $92, ?$ \\
\hline $\begin{array}{l}\text { bereinigter } \\
\text { erklärter } \\
\text { Varianzan- } \\
\text { teil }\end{array}$ & 45,5 & 49,7 & 45.2 \\
\hline
\end{tabular}

wo (von der 3. und den beiden letzten Zeilen abgesehen) in jeder Zelle zuerst der unstandardisierte Regressionskoeffizient, dann dessen Signifikanzniveau, dann der standardisierte Regressionskoeffizient angegeben ist;

ein Signifikanzniveau von 0,00 durch Abrundung eines kleineren Wertes als 0,0049 entstanden ist; der standardisierte Regressionskoeffizient in der 3. Zeile den Einfluß des wirtschaftlichen Entwicklungsniveaus erfaßst (vgl. Jagodzinski/Weede 1981 zur Berechnung und Interpretation).

Länder erfaßt ${ }^{12}$. In Tabelle 3 ist die Datenbasis nicht mehr 50, sondern 65. Der kurvilineare Zusammenhang zwischen BSPC und Einkommensverteilung ist jetzt signifikant. Der Effekt der Sekundareinschulungsquote bleibt hoch

12 In der Gleichung für den Anteil der obersten $20 \%$ ist $\mathbf{N}$ sogar 67. In Tab. 3 sind im Gegensatz zu den Tab. 1 und 2 keine impliziten Schätzungen von fehlenden Daten durch ,pairwise deletion' vorgenommen worden. Würde man fehlende Daten bei einzelnen Ländern und unabhängigen Variablen hinnehmen, dem Datensatz Bangladesh, Hongkong, Südafrika und die Schweiz hinzufügen und mit ,pairwise deletion' arbeiten, dann wären die Ergebnisse ähnlich wie in Tab. 3. Nur die Effekte des militärischen Partizipationsgrades wären stärker. signifikant und stark ${ }^{13}$. Der Effekt des militärischen Partizipationsgrades überspringt in zwei von drei Gleichungen das 5\%-Signifikanzniveau, in der dritten zumindest noch das $10 \%$-Niveau.

\section{Abschließende Diskussion}

Nach Lenski (1973) sollte die Einkommensverteilung vor allem durch die Machtverteilung erklärt werden. Wer mächtig ist, wird schon entsprechende Einkommen durchsetzen. Bornschier und Ballmer-Cao $(1979,1980)$ haben versucht, diesen Grundgedanken so auszuarbeiten, daß er in einem international vergleichenden Untersuchungsplan geprüft werden kann. Nach ihrer Auffassung kann der Zusammenhang zwischen Macht- und Einkommensverteilung so präzisiert werden: Je weiter die Bürokratisierung fortschreitet, je geringer die Landbesitzkonzentration, je größer die Drohstärke der Lohnabhängigen und je größer die Staatsquote, desto egalitärer wird die Einkommensverteilung sein.

Die hier vorgelegte Studie ist zunächst einmal ein Replikationsversuch, der sich nur in ausgewählten verfahrenstechnischen Fragen wie Zeitanpassung, Behandlung fehlender Datenangaben und (unserem Verzicht auf) Rekodierung mit Informationsverlust von der Studie Bornschiers und Ballmer-Caos unterscheidet. Dabei wollen wir nicht behaupten, daß unsere verfahrenstechnischen Entscheidungen besser sind als die von Bornschier und Ballmer-Cao (1979, 1980), sondern nur, daß sie annähernd ebenso gut oder ebenso fragwürdig sind und daß die inhaltliche Interpretation von Ergebnissen eine gewisse Unempfindlichkeit gegenüber in verschiedener Weise fragwürdigen, aber so oder so unvermeidbaren technischen Entscheidungen voraussetzt.

Nach diesem Kriterium können wir von Bornschiers und Ballmer-Caos $(1979,1980)$ Befunden nur einen einzigen bestätigen: Je geringer die Landbesitzkonzentration, desto größer die

13 Dieses Ergebnis widerspricht nicht dem Befund von Weede und Tiefenbach $1981 \mathrm{~b}$. Dort haben wir nur globale Einschulungsquoten verwendet, die nicht zwischen Primär- und Sekundarstufe unterscheiden. 
Gleichheit der Einkommensverteilung. Im Gegensatz zu Bornschier und Ballmer-Cao bezweifeln wir allerdings, ob dieser Zusammenhang überall gleich stark ist. Wahrscheinlich variiert er mit dem Agraranteil am BSP. Dieses Problem haben wir hier allerdings nicht weiterverfolgt.

Die unterschiedlichen Ergebnisse bei der Drohstärke der Lohnabhängigen scheinen vorwiegend an der Rekodierung mit Informationsverlust bei Bornschier und Ballmer-Cao $(1979,1980)$ und der darauf folgenden Schätzung etlicher Werte und an unserem Verzicht auf diese Prozeduren zu liegen. Der positive Effekt von Streiks mit großer Beteiligung bzw. von Drohstärke der Lohnabhängigen kann zumindest nicht mehr als empirisch bestätigt gelten. Je nach verfahrenstechnischen Entscheidungen gibt es Evidenz dafür oder dagegen.

Bürokratisierung und Staatsquote bewähren sich nur so lange als Prädiktoren der Einkommensverteilung, wie man empirisch besser abgesicherte Prädiktoren aus anderen theoretischen Ansätzen vernachlässigt: den ökonomischen Entwicklungsgrad, der kurvilinear mit der Verteilung zusammenhängt, die Sekundareinschulungsquote und den militärischen Partizipationsgrad. Sofern es im Rahmen dieser Studie Anhaltspunkte dafür gibt, daß die Machtverteilung die Einkommensverteilung beeinflußt, dann so: Nicht Bürokratisierung oder Ausweitung der Staatstätigkeit, sondern eine Zunahme des militärischen Partizipationsgrads trägt zur Egalisienung der Einkommensverteilung bei.

\section{Anhang}

Liste der in Tab. 3 berücksichtigten Länder mit Russett-Singer-Small-Code:

$\begin{array}{llll}002 & \text { USA } & 434 & \text { Benin (Dahomey) } \\ 020 & \text { Canada } & 436 & \text { Niger } \\ 051 & \text { Jamaica } & 437 & \text { Ivory Coast } \\ 052 & \text { Trinidad and Tobago } & 451 & \text { Sierra Leone } \\ 070 & \text { Mexico } & 452 & \text { Glana } \\ 091 & \text { Honduras } & 475 & \text { Nigeria } \\ 092 & \text { El Salvador } & 483 & \text { Chad } \\ 093 & \text { Nicaragua } & 501 & \text { Kenya } \\ 094 & \text { Costa Rica } & 510 & \text { Tanzania } \\ 095 & \text { Panama } & 551 & \text { Zambia } \\ 100 & \text { Colombia } & 553 & \text { Malawi } \\ 101 & \text { Venezuela } & 580 & \text { Madagascar } \\ 130 & \text { Ecuador } & 600 & \text { Morocco }\end{array}$

$\begin{array}{llll}135 & \text { Peru } & 616 & \text { Tunisia } \\ 140 & \text { Brazil } & 625 & \text { Sudan } \\ 145 & \text { Bolivia } & 630 & \text { Iran } \\ 155 & \text { Chile } & 640 & \text { Turkey } \\ 160 & \text { Argentina } & 645 & \text { Iraq } \\ 165 & \text { Uruguay } & 651 & \text { Egypt } \\ 200 & \text { United Kingdom } & 660 & \text { Lebanon } \\ 210 & \text { Netherlands } & 666 & \text { Israel } \\ 220 & \text { France } & 713 & \text { Taiwan } \\ 230 & \text { Spain } & 732 & \text { S. Korea } \\ 255 & \text { W. Germany } & 740 & \text { Japan } \\ 305 & \text { Austria } & 750 & \text { India } \\ 325 & \text { Italy } & 770 & \text { Pakistan } \\ 345 & \text { Yugoslavia } & 775 & \text { Burma } \\ 350 & \text { Greece } & 780 & \text { Sri Lanka (Ceylon) } \\ 375 & \text { Finland } & 800 & \text { Thailand } \\ 380 & \text { Sweden } & 820 & \text { Malaysia } \\ 385 & \text { Norway } & 840 & \text { Plitippines } \\ 390 & \text { Denmark } & 850 & \text { Indonesia } \\ 433 & \text { Senegal } & 900 & \text { Australia } \\ & & 920 & \text { New Zealand } \\ & & & \end{array}$

Die Daten sind im allgemeinen Ballmer-Cao und Scheidegger (1979) entnommen. Ausnahmen: Die Verteilungsdaten für Israel und Sudan stammen aus Paukert (1973: 114-115), für Nicaragua und Iran (nur für dic obersten 20\%) aus Chenery und Syrquin (1975: 196 197). Die BSPC-Angabe für Lebanon entstammt Taylor und Hudson (1972: 316).

\section{Literatur:}

Ahluwalia, M.S., 1976a: Income Distribution and Development. American Economic Review 66: 128 135.

Ahluwalia, M.S., 1976b: Inequality, Poverty and Development. Journal of Development liconomics 3: $307-342$.

Andreski, S., 1968: Military Organization and Socicty. Stanford, Calif.: Stanford University Press.

Ballmer-Cao, T.-H./Scheidegger, J., 1979: Compendium of Data for World System Analysis. Zürich: Soziologisches Institut der Universität.

Bornschier, V. (Hrsg.), 1980: Multinationale Konzerne, Wirtschaftspolitik und nationale Entwicklung im Weltsystem. Frankfurt: Campus.

Bornschier, V., $1981 \mathrm{a}$ : Arbcitsteilung, struk turelle Mobilität und Klassenbildung. Eine theoretische Perspektive für die Mobilitätsforschung. Zeitschrift für Soziologie 10: 117-132.

Bornschier, V., 1981 b: Weltwirtschaft, Wachstum und Verteilung. Schwcizerische Zeitschrift für Soziologie 7: 129-136.

Bornschier, V., 1981c: Comment (on Weede and Tiefenbach 1981a). International Studies Quarterly 25: 283-288.

Bornschier, V./Ballmer-Cao, T.-H., 1979: Income Inequality: A Cross-National Study of the Relationship Between MNC-Penetration, Dimensions of the Power Structure and Income Distribution. American Sociological Review 44: 487-506. Deutsche Fassung in Bornschier 1980: 213-240. 
Chenery, H./Syrquin, M., 1975: Patterns of Development 1950-1970. London: Oxford University Press.

Evans, P.B./Timberlake, M., 1980: Dependence, Inequality, and the Growth of the Tertiary. American Sociological Review 45: $531-552$.

Goldberger, A.S., 1970: On Boudon's Method of Linear Causal Analysis. American Sociological Review 35: 97-101.

Hayek, F.A. von, 1971: Die Verfassung der Freiheit. Tübingen: Mohr.

Jagodzinski, W./Weede, E., 1980: Weltpolitische und ökonomische Determinanten einer ungleichen Einkommensverteilung. Zeitschrift für Soziologie 9: $132-148$

Jagodzinski, W./Weede, E., 1981: Testing Curvilinear Propositions by Polynomial Regression with Particular Reference to the Interpretation of Standardized Solutions. Quality and Quantity 15: 447-463.

Kuznets, S., 1963: Quantitative Aspects of the Economic Growth of Nations. VIII: The Distribution of Income by Size. Economic Development and Cultural Change 11: 1-80.
Lenski, G., 1973: Macht und Privileg. Frankfurt: Suhrkamp.

McKenzie, R.B./Tullock, G., 1978: Modern Political Economy. Tokyo: McGraw-Hill Kogakusha.

Paukert, F., 1973: Income Distribution at Different Levels of Development. International Labour Review 108: 97-125.

Taylor, C.L./Hudson, M.C., 1972: World Handbook of Political and Social Indicators. 2nd ed. New Haven, Conn.: Yale University Press.

Weede, E., 1980: Beyond Misspecification in Sociological Analyses of Income Inequality. American Sociological Review 45: 497-501.

Weede, E., 1981: Militär, Multis und Wirtschaft. Schweizerische Zeitschrift für Soziologie 7: 113127.

Weede, E./Tiefenbach, H., 1981a: Some Recent Explanations of Income Inequality. International Studies Quarterly 25: 255-282 and 289-293.

Weede, E./Tiefenbach, H., 1981 b: Correlates of the Size Distribution of Income in Cross-National Analysis. Journal of Politics 43: 1029-1041. 